\title{
Effect of Adaptation Gain in Model Reference Adaptive Controlled Second Order System
}

\author{
Pankaj Swarnkar \\ Dept of Electrical Engineering \\ Maulana Azad National Institute of \\ Technology \\ Bhopal, India
}

\author{
Shailendra Jain \\ Dept of Electrical Engineering \\ Maulana Azad National Institute of \\ Technology \\ Bhopal, India
}

\author{
R. K. Nema \\ Dept of Electrical Engineering \\ Maulana Azad National Institute of \\ Technology \\ Bhopal, India
}

\begin{abstract}
Adaptive control involves modifying the control law used by the controller to cope with the fact that the parameters of the system being controlled change drastically due to changes in environmental conditions or in system itself. This technique is based on the fundamental characteristic of adaptation of living organism. The adaptive control process is one that continuously and automatically measures the dynamic behavior of a plant, compares it with the desired output and uses the difference to vary adjustable system parameters or to generate an actuating signal in such a way so that optimal performance can be maintained regardless of system changes. The nature of the adaptation mechanism for controlling the system performance is greatly affected by the value of adaptation gain. It is observed that for the lower order systems, wide range of adaptation gain can be used to study the system's performance. As the order of the system increases the applicable range of adaptation gain becomes narrow. This paper deals with application of model reference adaptive control (MRAC) scheme to second order systems with different values of adaptation gain. The rule which is used for this application is the MIT rule. Simulation is done in MATLAB and simulink and the results are compared for varying adaptation mechanisms due to variation in adaptation gain.
\end{abstract}

Keywords-- model reference adaptive control, MIT rule, adaptation mechanism, adaptation gain

\section{INTRODUCTION}

A control system is in the broadest sense, an interconnection of the physical components to provide a desired function, involving some type of control action with it. The requirement of high performance control systems for industrial applications has produced great research efforts for the application of modern control theory and, in particular, adaptive control. Adaptive control is a rather recent class of control technique, although research in adaptive control has a long and vigorous history. In 1950s, it was motivated by the problem of designing autopilots for aircraft operating at wide range of speeds and altitudes. Consequently, gain scheduling based on some auxiliary measurements of air speed was adapted. Kalman [1] developed the concept of a general self tuning regulator with explicit identification of the parameters of a linear, single-input, single-output system and he used these parameters' estimation to update an optimal linear quadratic controller, and other researchers (most significantly Lyapunov and Parks) further advanced the concept. Nowadays the adaptive control schemes are making their place where the conventional control system is not able to cope-up with the situation, like:

- loads, inertias and other forces acting on the system, change drastically.

- possibility of unpredictable and sudden faults.

- $\quad$ possibility of frequent or unanticipated disturbances.

The conventional PID controllers with fixed gain are unable to cope up with the problems discussed above. However, advanced fuzzy PID controllers [2-9] have been recently developed to deal with such problems for electrical and mechanical systems. Also the concept of neural network has been applied [10-12] in order to enhance the dynamic characteristics of the PID controllers. Still, to obtain the complete adaptive nature, specific adaptive control techniques are needed. Adaptive control changes the control algorithm coefficients in real time to compensate for variations in environment or in the system itself. It also varies the system transfer function according to situation. In practical cases, a model reference adaptive control system is generally best implemented with a digital computer, owing to the complexity of the controller.

Out of many adaptive control schemes, this paper mainly deals with the model reference adaptive control approach based on MIT rule [13-17]. In MRAC [13-24], the output response is forced to track the response of a reference model irrespective of plant parameter variations. The controller parameters are adjusted to give a desired closed-loop performance. Here the controller parameters are estimated to cause the desired change in plant transfer function so that its performance can be made similar to the reference model. Apart from the MIT rule there are some other design techniques in Model Reference Adaptive Control system, like Lyapunov theory [23-24] and the theory of augmented error [25]. In this paper the emphasis is given to MIT rule only for developing the MRAC scheme. The system response is calculated for different values of adaptation gain.

\section{MODEL REFERENCE ADAPTIVE CONTROL}

This technique of adaptive control falls under the category of Non-dual adaptive control. A reference model describes the 
system's performance. The adaptive controller is then designed to force the system (or plant) to behave like the reference model. Model output is compared to the actual output, and the difference is used to adjust feedback controller parameters.

MRAS has two loops: an inner loop (or regulator loop) that is an ordinary control loop consisting of the plant and the regulator, and an outer (or adaptation) loop that adjusts the parameters of the regulator in such a way as to drive the error between the model output and plant output to zero, as shown in Figure 1 .

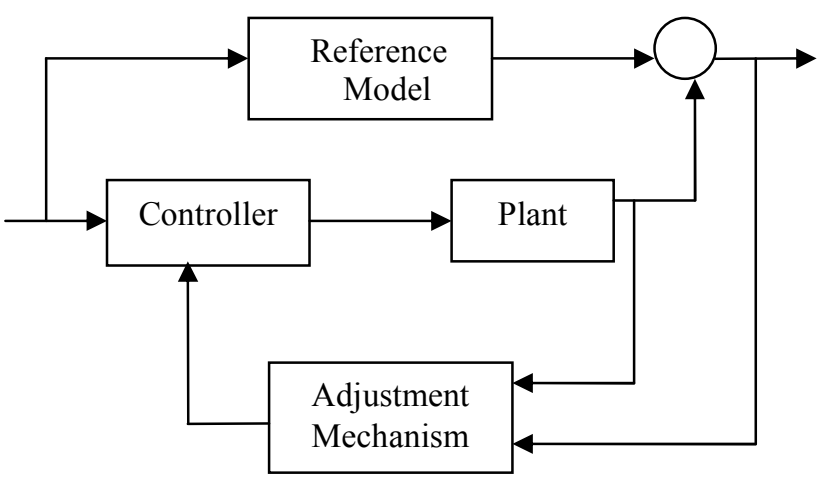

Fig. 1. Model Reference Adaptive Controller

\section{COMPONENTS OF MODEL REFERENCE ADAPTIVE CONTROLLER}

Reference Model: It is used to specify the ideal response of the adaptive control system to external command. It should reflect the performance specifications in control tasks. The ideal behavior specified by the reference model should be achievable for the adaptive control system. In this paper the critically damped second order system is taken as the reference model.

Controller: It is usually parameterized by a number of adjustable parameters. In this paper, two parameters $\theta_{1}$ and $\theta_{2}$ are used to define the controller law. The control law is linear in terms of the adjustable parameters (linear parameterization). Adaptive controller design normally requires linear parameterization in order to obtain adaptation mechanism with guaranteed stability and tracking convergence. The values of these control parameters are mainly dependent on adaptation gain which in turn changes the control algorithm of adaptation mechanism.

Adaptation Mechanism: It is used to adjust the parameters in the control law. Adaptation law searches for the parameters such that the response of the plant which should be same as the reference model. It is designed to guarantee the stability of the control system as well as conversance of tracking error to zero. Mathematical techniques like MIT rule, Lyapunov theory and augmented error theory can be used to develop the adaptation mechanism. In this paper the MIT rule is used for this purpose.

\section{THE MIT RULE}

This rule was developed in Massachusetts Institute of Technology and is used to apply the MRAC approach to any practical system. In this rule the cost function or loss function is defined as

$$
F(\theta)=\mathrm{e}^{2} / 2
$$

Where, e is the output error and is the difference of the output of the reference model and the actual model, while $\theta$ is the adjustable parameter known as the control parameter.

In this rule the parameter $\theta$ is adjusted in such a way so that the loss function is minimized. Therefore, it is reasonable to change the parameter in the direction of the negative gradient of $F$, that is:

$$
\begin{aligned}
& \mathrm{d} \theta / \mathrm{dt}=-\gamma \partial F / \partial \theta \\
& =-\gamma \mathrm{e} \partial \mathrm{e} / \partial \theta
\end{aligned}
$$

The partial derivative term $\partial \mathrm{e} / \partial \theta$, is called the sensitivity derivative of the system. This shows how the error is dependent on the adjustable parameter, $\theta$. There are many alternatives to choose the loss function $F$. For example, it can also be taken as mode of error. Similarly $\mathrm{d} \theta / \mathrm{dt}$ can also have different relations for different applications.

Sign-sign algorithm:

$$
\mathrm{d} \theta / \mathrm{dt}=-\gamma \operatorname{sign}(\partial \mathrm{e} / \partial \theta) \text { sign } \mathrm{e}
$$

Or it may be chosen as :

$$
\mathrm{d} \theta / \mathrm{dt}=-\gamma(\partial \mathrm{e} / \partial \theta) \text { sign } \mathrm{e}
$$

Where sign $\mathrm{e}=1$ for $\mathrm{e}>0$

$$
\begin{aligned}
& =0 \text { for } \mathrm{e}=0 \\
& =-1 \text { for } \mathrm{e}<0
\end{aligned}
$$

In some industrial applications it is found that the choice of adaptation gain is critical and its value depends on the signal levels. So MIT rule has to be modified as follows:

$$
\mathrm{d} \theta / \mathrm{dt}=-\gamma \zeta \mathrm{e}
$$

Where $\zeta=\partial \mathrm{e} / \partial \theta$

Also $d \theta / d t=-\gamma \zeta$ e $/\left(\beta+\zeta^{\mathrm{T}} \zeta\right)$

Where $\beta>0$ is introduce to avoid the zero division when $\zeta^{\mathrm{T}}$ $\zeta$ is small. This paper uses the MIT rule defined by equations (1), (2) and (3) for developing the control law.

\section{MATHEMATICAL MODELLING}

In this paper Model Reference Adaptive Control Scheme is applied to a second order system using MIT rule. It is a well known fact that an under damped second order system is oscillatory in nature. If oscillations are not decaying in a limited time period then they may cause system instability. So, for stable operation, maximum overshoot must be as low as possible (ideally zero). This can automatically reduce the transient period of the system and improve the system's performance. A critically damped second order system gives a characteristic without any oscillations and this characteristic is similar to the first order system. But it is not feasible to achieve such system practically. In this paper a second order under damped system with large settling time, very high maximum overshoot and with intolerable dynamic error is taken as a plant. The object is to improve the performance of this system by using adaptive control scheme. For this purpose, a critically 
damped system is taken as the reference model. Let the second order system be described by:

$$
d^{2} y / d t^{2}=-a(d y / d t)-b y+b u
$$

Let $a=8$ and $b=600$

Where $\mathrm{y}$ is the output of plant (second order under damped system) and $u$ is the controller output or manipulated variable. The transfer function can be written as:

$$
\mathrm{Y}(\mathrm{s}) / \mathrm{U}(\mathrm{s})=600 /\left(\mathrm{s}^{2}+8 \mathrm{~s}+600\right)
$$

Similarly the reference model is described by:

$$
d^{2} y_{m} / d t^{2}=-a_{m} d y_{m} / d t-b_{m} y_{m}+b_{m} r
$$

Take $\mathrm{a}_{\mathrm{m}}=8$ and $\mathrm{b}_{\mathrm{m}}=16$

Where $y_{m}$ is the output of reference model (second order critically damped system) and $r$ is the reference input (unit step input). The transfer function is written as:

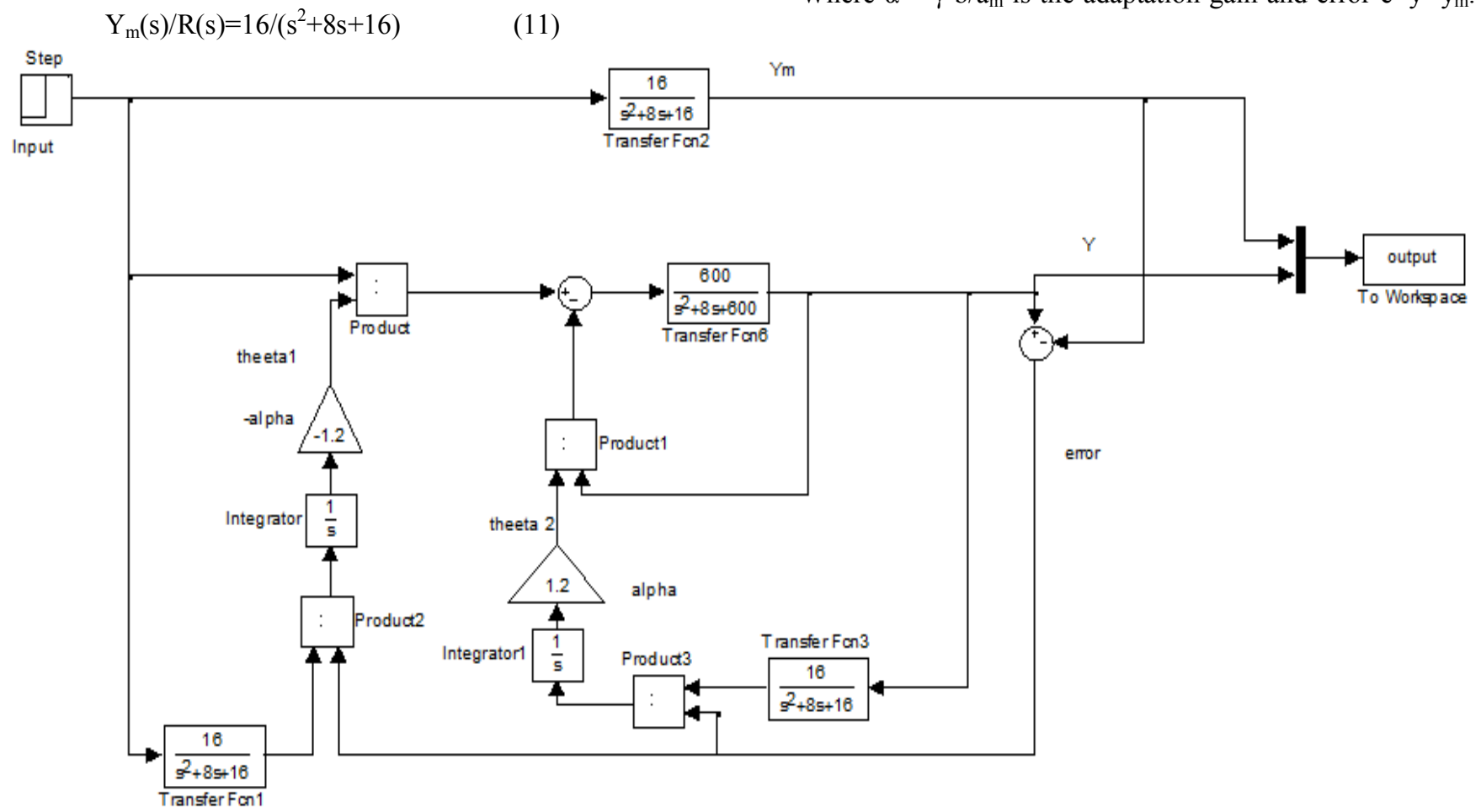

Fig. 2. Simulated model for MIT rule

\section{Simulation AND RESUltS}

In this paper the model reference adaptive control model is simulated in MATLAB and simulink. Here the MIT rule is applied to the second order system. The simulation model is shown in Figure 2.

The time response characteristics for the plant and the reference model are shown in Figure 3, with out using any control technique. It can be observed that the characteristic of the plant is oscillatory with high value of overshoot and undershoot whereas the characteristic of the reference model is smooth without any oscillation. There is a large dynamic error between these two and this error has to be reduced to zero by using model reference adaptive control scheme.
Here the object is to compare the actual output (y) and the reference output $\left(\mathrm{y}_{\mathrm{m}}\right)$ and, by applying model reference adaptive control scheme, to improve the overall output. Let the controller be described by the law:

$$
\mathrm{u}(\mathrm{t})=\theta_{1} \mathrm{r}(\mathrm{t})-\theta_{2} \mathrm{y}(\mathrm{t})
$$

The controller parameters are chosen as:

$\theta_{1}=b_{m} / b$ and $\theta_{2}=\left(a_{m}-a\right) / b$

The update rule for the controller parameters using MIT rule is described by:

$$
\begin{aligned}
\mathrm{d} \theta_{1} / \mathrm{dt} & =-\gamma \text { e } \partial \mathrm{e} / \partial \theta_{1} \\
& =-\gamma \mathrm{e}\left[\mathrm{b} \mathrm{r} /\left(\mathrm{p}+\mathrm{a}_{\mathrm{m}}\right)\right] \\
& =-\alpha \mathrm{e}\left[\mathrm{a}_{\mathrm{m}} \mathrm{r} /\left(\mathrm{p}+\mathrm{a}_{\mathrm{m}}\right)\right]
\end{aligned}
$$

and $d \theta_{2} / d t=-\alpha e\left[a_{m} y /\left(p+a_{m}\right)\right]$

Where $\alpha=\gamma b / a_{m}$ is the adaptation gain and error $\mathrm{e}=\mathrm{y}-\mathrm{y}_{\mathrm{m}}$. 


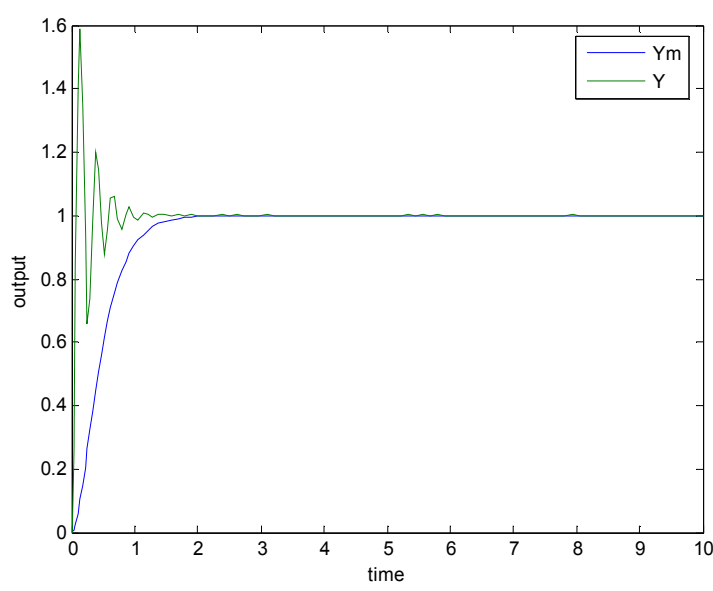

Fig. 3. Time response curve (without any control)

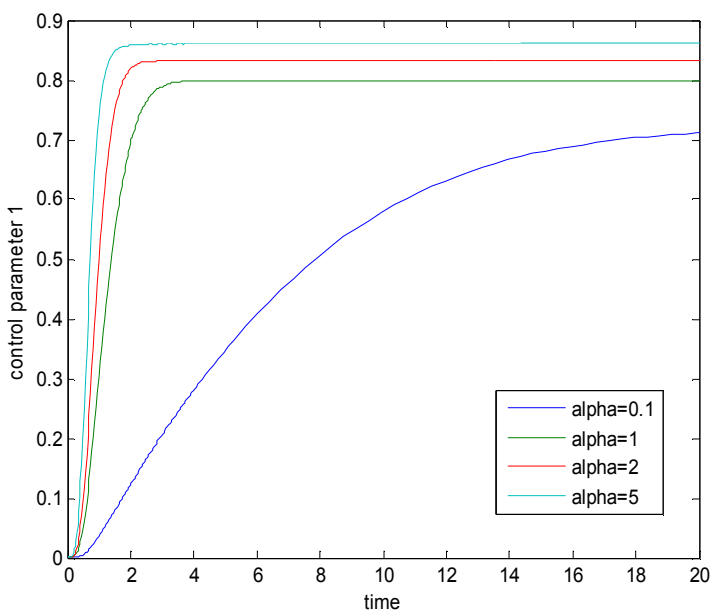

Fig. 4. Variation of control parameter $\theta_{1}$ for different values of $\alpha$

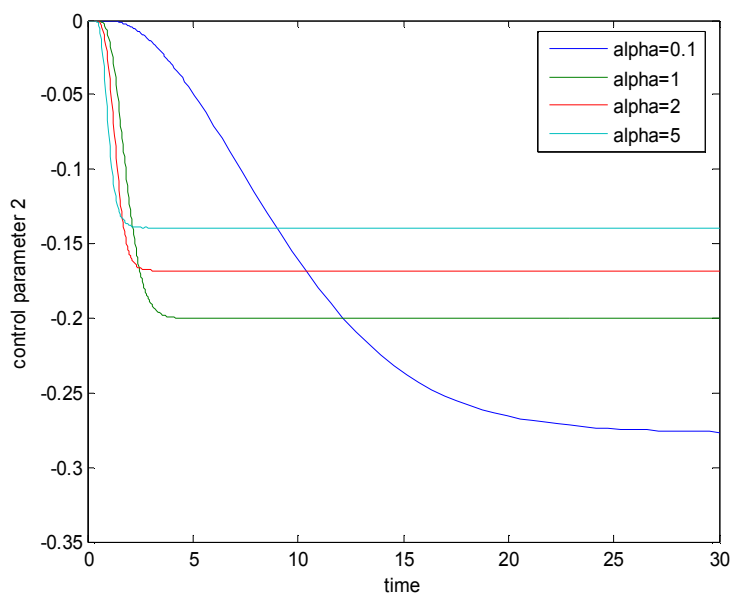

Fig. 5. Variation of control parameter $\theta_{2}$ for different values of $\alpha$

Now by using equation (13) the estimated plant parameters come out to be

$$
\mathrm{b}^{\prime}=\mathrm{b}_{\mathrm{m}} / \theta_{1} \quad \text { and } \quad \mathrm{a}^{\prime}=\mathrm{a}_{\mathrm{m}}-\mathrm{b}^{\prime} \theta_{2}
$$

Figure 6 shows the output characteristic (response curve) for the system with Model Reference Adaptive Control scheme. It can be seen that the characteristic of the plant has improved drastically. There is no oscillation in the system response. Also the effect of adaptation gain is shown in Figure 6 . The better response can be achieved for the higher values of adaptation gain. The best result is obtained for $\alpha$ equal to 5, for the selected range of adaptation gain.

Finally the error curve is shown in Figure 7. The dynamic error is reducing with the increment in adaptation gain. It implies that by MRAC the plant output is following the desired output which was the main aim behind the control action.

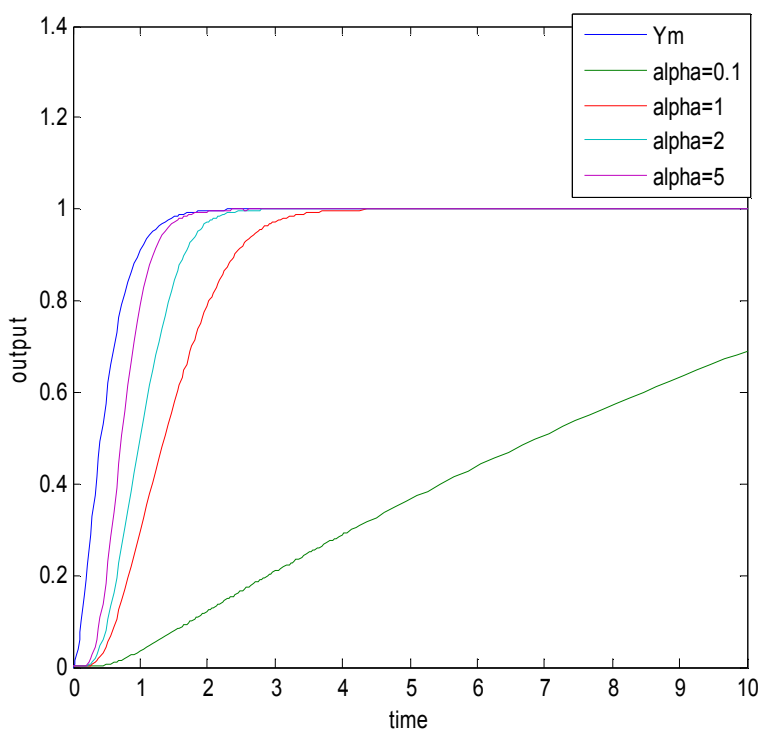

Fig. 6. effect of $\alpha$ on time response characteristic

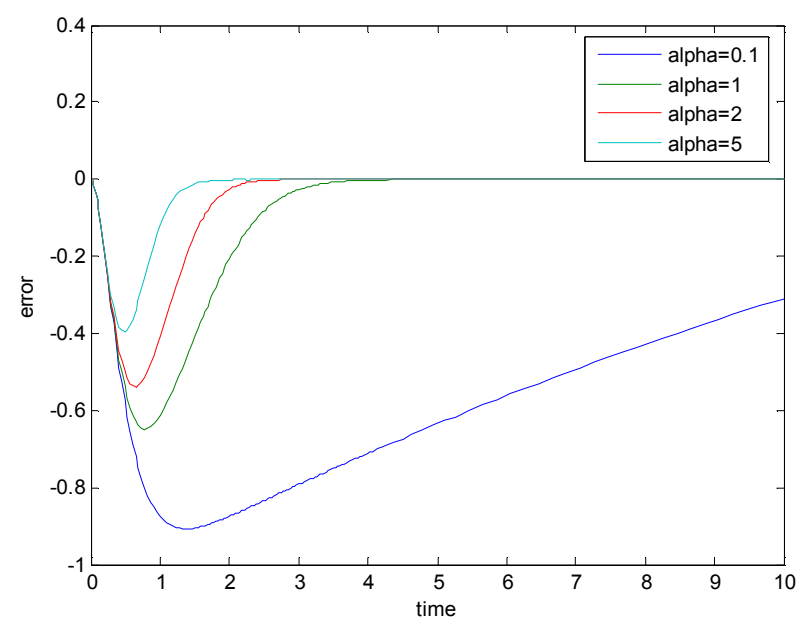

Fig. 7. Error versus time curve

\section{CONCLUSION}

As compared to conventional fixed gain controllers (PID Controllers), the adaptive controllers are very effective to 
handle the situations where the parameter variations and environmental changes are frequent. This fact is demonstrated clearly in the results. Also the use of PI control mainly improves the steady state performance by reducing the steady state error whereas PD controller improves the transient period by reducing the maximum overshoot. But in case of adaptive control the entire dynamic characteristic of the system is improved. Here the controller parameters are adjusted to give a desired closed-loop performance throughout the working of the system. The adaptive controller maintains constant dynamic performance in the presence of unpredictable and immeasurable variations. Application of MIT rule in a model reference adaptive controller results clearly to an improvement in system's performance. The tracking error is tending to zero and the performance of the actual plant is approaching the performance of the reference model. Table I shows that without any control, plant performance was very poor with very high values of overshoot and undershoot. Whereas the application of MRAC by using MIT rule reduces the values of overshoot and undershoot directly to zero. This paper mainly emphasis on the effect of adaptation gain on the system's performance. Table I shows that with the increment in adaptation gain the settling time is reducing. Also the control parameters vary in the direction to improve the system performance with the increment in adaptation gain. But beyond the chosen range of adaptation gain $(0.1<\alpha<5)$, system performance is very poor. The system may even become unstable for the wrong choice of adaptation gain. Therefore, it is shown that for suitable values of adaptation gain, the MIT rule can make the plant output as close as possible to the reference model in model reference adaptive control scheme.

TABLE I

TIME RESPONSE SPECIFICATIONS WITH AND WITHOUT MRAC FOR DIFFERENT ADAPTATION GAIN

\begin{tabular}{|c|c|c|c|c|c|}
\hline & \multirow{2}{*}{$\begin{array}{l}\text { Without any } \\
\text { control }\end{array}$} & \multicolumn{4}{|c|}{ With MRAC } \\
\hline & & $\alpha=0.1$ & $\alpha=1$ & $\alpha=2$ & $\alpha=5$ \\
\hline Peak Time (second) & 0.135 & 0 & 0 & 0 & 0 \\
\hline $\begin{array}{c}\text { Maximum } \\
\text { Overshoot (\%) } \\
\end{array}$ & $60 \%$ & 0 & 0 & 0 & 0 \\
\hline Undershoot (\%) & $34 \%$ & 0 & 0 & 0 & 0 \\
\hline $\begin{array}{l}\text { Settling Time } \\
\text { (second) }\end{array}$ & 1.7 & 28 & 4 & 3.6 & 2 \\
\hline$\theta 1$ & $\ldots \ldots$ & 0.722 & 0.8 & 0.832 & 0.86 \\
\hline$\theta 2$ & f....... & -0.227 & -0.2 & -0.168 & -0.14 \\
\hline
\end{tabular}

\section{REFERENCES}

[1] D. Simon, "Kalman filtering with state constraints- a survey of linear and nonlinear algorithm", Control Theory and Application, IET, Vol. 4, No. 8, pp. $1303-1318,2010$.

[2] Rey-Chue Hwang, Huang-Chu Huang, Wei-Shen Chi, “A New Fuzzy PID-Like Controller", IEEE International Conference on Systems, Man and Cybernetics, Nashville, Vol. 5, 2000.

[3] K. S. Tang, Kim Fung Man, Guanrong Chen, Sam Kwong, “An Optimal Fuzzy PID Controller", IEEE Transactions on Industrial Electronics, Vol. 48, No. 4, pp. 757-765, 2001.

[4] K. L. Lo and M. O. Sadegh, "Systematic Method for the Design of A Full-Scale Fuzzy PID Stability Controller for SVC to Control Power System", IEEE Transaction on Generation Transmission and Distribution Vol. 150, No. 3, pp. 297 - 304, 2003.

[5] E. M. Jafarov, M. N. A. Parlakçı, and Y. Istefanopulos, "A New Variable Structure PID-Controller Design for Robot Manipulators", IEEE Transactions on Control Systems Technology, Vol. 13, No. 1, pp. $122-130,2005$

[6] Tae-Yong Choi, Kap-Ho Seo, Jin-Ho Shinü, Ju-Jang Lee, "The Hybrid SOF-PID Controller for A MIMO Nonlinear System", Proceedings of the 2005 IEEE/ASME, International Conference on Advanced Intelligent Mechatronics Monterey, California, USA, pp. 825 - 830, 2005.

[7] B. Jia, G. Ren, G. Long, "Design and Stability Analysis of Fuzzy Switching PID Controller", 6th World Congress on Intelligent Control and Automation, Dalian, China, pp. 3934 - 3938, 2006.

[8] A. Rubaai, M. J. Castro-Sitiriche, A. Ofoli, "DSP-Based Implementation of Fuzzy-PID Controller Using Genetic Optimization for High Performance Motor Drives", IEEE International Conference on Industry Applications, New Orleans, LA, pp. 1649 - 1656, 2007.
[9] A. Rubaai, M. J. Castro-Sitiriche, A. Ofoli, "DSP-Based Laboratory Implementation of Hybrid Fuzzy-PID Controller Using Genetic Optimization for High-Performance Motor Drives", IEEE Transactions on Industry Applications, Vol. 44, No. 6, pp. 1977 - 1986, 2008.

[10] D. Sun, J. Meng, “A Single Neuron PID Controller Based PMSM DTC Drive System Fed by Fault Tolerant 4-Switch 3-Phase Inverter”, IEEE International Conference on Industrial Electronics and Applications, Singapore, pp. 1-5, 2006.

[11] J. Yao, L. Wang, C. Wang, Z. Zhang, P. Jia, "ANN-Based PID Controller for An Electro-Hydraulic Servo System”, IEEE International Conference on Automation and Logistics, Qingdao, China, pp. $18-22$, 2008.

[12] Xue-Kui Wang, Xu-Hong Yang, Gang Liu, Hong Qian, “Adaptive Neuro-Fuzzy Inference System PID Controller for SG Water Level of Nuclear Power Plant", Proceedings of The Eighth International Conference on Machine Learning and Cybernetics, Baoding, pp. 567 $572,2009$.

[13] K. Benjelloun, H. Mechlih, E. K. Boukas, "A Modified Model Reference Adaptive Control Algorithm for DC Servomotor", Second IEEE Conference on Control Applications, Vancouver, B. C., Vol. 2, pp. $941-946,1993$

[14] M. S. Ehsani, "Adaptive Control of Servo Motor by MRAC Method", IEEE International Conference on Vehicle, Power and Propulsion, Arlington, TX, pp. $78-83,2007$.

[15] M. Kirar, P. Swarnkar, S. Jain, R. K. Nema, "Comparative Study of Conventional and Adaptive Schemes for DC Servomotors”, International Conference on Energy Engineering ICEE, Puducherry, India, 2009.

[16] P. Swarnkar, S. Jain, R. K. Nema, "Application of Model Reference Adaptive Control Scheme To Second Order System Using MIT Rule", International Conference on Electrical Power and Energy Systems (ICEPES-2010), MANIT, Bhopal, India, 2010.

[17] P. Swarnkar, S. Jain, R. K. Nema, "Effect of Adaptation Gain on System Performance for Model Reference Adaptive Control Scheme Using MIT 
Rule", International Conference of World Academy of Science, Engineering and Technology, Paris, FRANCE, 2010.

[18] Pin-Yan Tsai, Hung Chu Huang, Yu-Ju-Chen, Rey-Chue Hwang, "The Model Reference Control by Auto Tuning PID-Like Fuzzy Controller", International Conference on Control Applications, Taipei, Taiwan, 2004.

[19] M. Cirrincione, M. Pucci, "An MRAS-Based Sensorless High Performance Induction Motor Drive With A Predictive Adaptive Model", IEEE Transaction on Industrial Electronics, Vol. 52, No. 2, pp. $532,2005$.

[20] Kuo-Ming Chang, "Model Reference Adaptive Control for Uncertain Systems With Sector-Like Bounded Nonlinear Inputs", International Conference on Control and Automation, Budapest, Hungary, 2005.

[21] M. T. Benchouia, A. Ghamri, M. E. H. Benbouzid, A. Golea, S. E. Zouzou, "Fuzzy Model Reference Adaptive Control of Power Converter for Unity Power Factor and Harmonics Minimization", International Conference on Electrical Machine and System, Seoul, Korea, 2007.

[22] T. John Koo, "Stable Model Reference Adaptive Fuzzy Control of A Class of Nonlinear Systems", IEEE Transactions on Fuzzy Systems, Vol. 9, No. 4, pp. 624-636, 2001.

[23] Yuan-Rui Chen Jie Wu, Norbert C. Cheung, "Lyapunov's Stability Theory-Based Model Reference Adaptive Control for Permanent Magnet Linear Motor Drives", $1^{\text {st }}$ IEEE International Conference on Power Electronics System and Applications, Hong Kong, China, pp. 260 - 266, 2004.

[24] Kuo-Kai Shyu, Ming-Ji Yang, Yen-Mo Chen, Yi-Fei Lin, "Model Reference Adaptive Control Design for A Shunt Active-Power-Filter System", IEEE Transactions on Industrial Electronics, Vol. 55, No. 1, pp. $97-106,2008$.

[25] K. J. Astrom, Bjorn Wittenmark, "Adaptive Control”, 2nd Ed. Pearson Education Asia, pp. 185-225, 2001.

[26] Pankaj Swarnkar, “Automatic Control System”, $3^{\text {rd }}$ Ed. Satya Prakashan, New Delhi, 2010.

\section{AUTHORS PROFILE}

Pankaj Swarnkar received the B.E. degree from Govt. Engineering College, Jabalpur, India in 1998 and the M.Tech degree from Maulana Azad National Institute of Technology, Bhopal, India in 2004. Currently he is an Assistant Professor in Maulana Azad National Institute of Technology, Bhopal and pursuing the $\mathrm{PhD}$ degree. His field of interest includes control system, network analysis and power systems. He is the author of five technical books.

S. K. Jain received the B.E. degree from Samrat Ashok Technological Institute, Vidisha, India, in 1990, the M.E. degree from SGSITS, Indore, India, in 1994, and the Ph.D. degree from the Indian Institute of Technology, Roorkee, India, in 2003. Currently he is an Assistant Professor in the Department of Electrical Engineering, National Institute of Technology, Bhopal, India. His research interests include power electronics, electric drives, active power filters, and high-power factor converters. Dr. Jain received the AICTE - Career Award for Young Teachers for the year 2003-2004.

R. K. Nema received the B.E. degree from Barkatullah University, Bhopal, India, in 1986, the M.E. degree from Barkatullah University, Bhopal, India, in 1992, and the Ph.D. degree from Bhopal University, India, in 2004. Currently he is an Associate Professor in the Department of Electrical Engineering, National Institute of Technology, Bhopal, India. His research interests include power electronics, electric drives and DSP. 